\title{
Treatment with the PARP inhibitor, niraparib, sensitizes colorectal cancer cell lines to irinotecan regardless of MSI/MSS status
}

\author{
Sybil M Genther Williams ${ }^{1 *}$, Apryle M Kuznicki ${ }^{2}$, Paula Andrade ${ }^{2}$, Brian M Dolinski ${ }^{1}$, Cem Elbi ${ }^{1,3}$, Ronan C O'Hagan ${ }^{1}$ \\ and Carlo Toniatti $i^{1,4}$
}

\begin{abstract}
Background: Cells with homologous recombination (HR) deficiency, most notably caused by mutations in the BRCA1 or BRCA2 genes, are sensitive to PARP inhibition. Microsatellite instability (MSI) accounts for 10-15\% of colorectal cancer (CRC) and is hypothesized to lead to HR defects due to altered expression of Mre11, a protein required for double strand break (DSB) repair. Indeed, others have reported that PARP inhibition is efficacious in MSI CRC.

Methods: Here we examine the response to niraparib, a potent PARP-1/PARP-2 inhibitor currently under clinical evaluation, in MSI versus microsatellite stable (MSS) CRC cell lines in vitro and in vivo. We compiled a large panel of MSI and MSS CRC cell lines and evaluated the anti-proliferative activity of niraparib. In addition to testing single agent cytotoxic activity of niraparib, we also tested irinotecan (or SN-38, the active metabolite of irinotecan) activity alone and in combination with niraparib in vitro and in vivo.

Results: In contrast to earlier reports, MSI CRC cell lines were not more sensitive to niraparib than MSS CRC cell lines, suggesting that the MSI phenotype does not sensitize CRC cell lines to PARP inhibition. Moreover, even the most sensitive MSI cell lines had niraparib EC50s greater than 10 fold higher than BRCA-deficient cell lines. However, MSI lines were more sensitive to SN-38 than MSS lines, consistent with previous findings. We have also demonstrated that combination of niraparib and irinotecan was more efficacious than either agent alone in both MSI and MSS cell lines both in vitro and in vivo, and that niraparib potentiates the effect of irinotecan regardless of MSI status.
\end{abstract}

Conclusions: Our results support the clinical evaluation of this combination in all CRC patients, regardless of MSI status.

\section{Introduction}

Poly (ADP-ribose) polymerase (PARP) enzymes are involved in repair of single strand DNA lesions using the base excision repair (BER) pathway. Inhibition of PARP enzymes induces persistence of single strand breaks (SSBs) which can cause double strand breaks (DSBs) when the SSBs are encountered by a replication fork. The development of PARP inhibitors as agents to treat cancers with homologous recombination (HR) defects is based on the idea that cells with defects in DSB repair, such as BRCAdeficient cells, are more dependent on PARP and BER to maintain genomic integrity [1,2]. Indeed, preclinical

\footnotetext{
*Correspondence: sybil_williams@merck.com

'Department of Oncology, Merck Research Laboratories, 33 Avenue Louis Pasteur, Boston, MA 02115, USA

Full list of author information is available at the end of the article
}

and clinical evidence have demonstrated that PARP inhibitors are synthetic lethal for tumors with mutations in the BRCA1 and BRCA2 genes and other genes involved in HR [1-5].

The instability of microsatellite repeated sequences, MSI, is found in tumors from the familial cancer syndrome hereditary nonpolyposis colorectal cancer (HNPCC) and in $10-15 \%$ of sporadic CRC. The MSI phenotype is a marker of an underlying mismatch repair (MMR) defect which stems from germline mutation in one of the MMR genes (principally $\mathrm{MLH1}$ or $\mathrm{MSH} 2$ ) or aberrant methylation of the $M L H 1$ promoter. One consequence of MSI is the reduced expression of the Mre11 protein resulting from mutation of the poly $(\mathrm{T}) 11$ repeat within intron 4 of human MRE11 [6]. Reduced expression of Mre11 is hypothesized 
to lead to defects in HR, due to Mre11's essential role in sensing DSBs and facilitating their repair [6-10].

PARP inhibition is effective in combination with irradiation and DNA-damaging agents [11]. In particular, PARP inhibitors have been shown to potentiate the effects of Topoisomerase 1 (Top 1) inhibitors both preclinically and clinically [12-17]. Top1 inhibition slows replication fork progression and induces the widespread formation of unusual replication intermediates, most notably reverse replication forks [12]. PARP activity is required for effective fork reversal, which limits the number of DSBs that result [12].

Irinotecan, a Top 1 inhibitor, is used as a therapy for $\mathrm{CRC}$ either alone or in combination with leucovorin and 5-Fluorouracil (5-FU). MSI is associated with increased sensitivity to irinotecan, both in vitro and in patients with advanced colon cancer $[9,18,19]$. The mechanism underlying this observation is not well understood.

Niraparib is a potent and selective orally available PARP-1/2 inhibitor [3]. In vitro and in vivo, niraparib displays outstanding monotherapy efficacy in a large panel of BRCA mutant cell lines with at least 10-fold selectivity over BRCA wild type cell lines [3]. In this study, the efficacy of niraparib was evaluated in the presence and absence of irinotecan in models of CRC with defects associated with the MSI phenotype as compared to MSS phenotype.

We demonstrate that the MSI phenotype does not overtly sensitize CRC cell lines to PARP inhibition and confirm that MSI CRC cell lines are more sensitive to SN-38 (active metabolite of irinotecan) than MSS cell lines. Niraparib potentiated the cytotoxic activity of irinotecan in both MSI and MSS CRC models. Our data suggests that both MSI and MSS patient populations will benefit from the combination of niraparib and irinotecan.

\section{Methods \\ Microsatellite repeat analysis}

DNA was extracted using standard methods from cells that were plated one day prior. Cell lines that were used in this study were described as being either MSI or MSS in previous publications [20-25]. The cell lines analyzed for MSI included: MSI- COLO205, DLD-1, HCT8, HCT15, HCT116, LOVO, LS411N, RKO, RKO-E6, SW48 and MSS-SW403, SW1417, WIDR. Primers used to amplify BAT-25 and BAT-26 were (BAT-25) 5'- 6FAM-TCG CCT CCA AGA ATG TAA GT-3' and 5'-TCT GCA TTT TAA CTA TGG CTC-3' (BAT-26) 5'-HEX- TGA CTA CTT TTG ACT TCA GCC-3' and 5'-AAC CAT TCA ACA TTT TTA ACC-3'. PCR amplification was performed with primers at $200 \mathrm{nM}$ each with $1 \mathrm{X}$ concentration of colorless GoTaq Flexi buffer (Promega Cat. No. M8305), $2 \mathrm{mM} \mathrm{MgCl} 2,0.2 \mathrm{mM}$ of each dNTP (PCR nucleotide Mix Promega cat. No. C1141), 1.25 u of GoTaq
DNA polymerase ( $5 \mathrm{u} / \mu \mathrm{l}$; Promega cat. No. M8305), $50 \mathrm{ng}$ of DNA, and nuclease free water to $50 \mu \mathrm{l}$. PCR conditions were: $95^{\circ} \mathrm{C}$ for 2 minutes followed by 35 cycles of $94^{\circ} \mathrm{C}$ for 1 minute, $55^{\circ} \mathrm{C}$ for 1 minute and $72^{\circ} \mathrm{C}$ for 1 minute, followed by a final extension of $72^{\circ} \mathrm{C}$ for 5 minutes and the $4^{\circ} \mathrm{C}$. To analyze the PCR products the following mix was prepared: $10 \mu \mathrm{lHi}$-Dri Formamide (Applied Biosystems Cat. No. 4311320) + $0.05 \mu \mathrm{l}$ GeneScan $500 \mathrm{LIZ}$ marker (Applied Biosystems Cat. No. 4322362). PCR product was diluted 1:400 in water. $1 \mu \mathrm{l}$ of PCR product was added to $10 \mu \mathrm{l}$ of Hi-Dri Formamide/Gene Scan $500 \mathrm{LIZ}$ marker mix and samples were heated at $95^{\circ} \mathrm{C}$ for 5 minutes. The fluorescently labeled PCR products were detected using the Sequencer 3730 DNA analyzer (Applied Biosystems) and analyzed using Peak Scanner software (Applied Biosystems).

\section{Western analysis}

$10 \mathrm{~cm}$ dishes were lysed with $50-150 \mu \mathrm{l}$ (depending on cell density) of boiling 1\% SDS Lysis Buffer [50 ml-10\% SDS $(5 \mathrm{ml}) 5 \mathrm{M} \mathrm{NaCl}(1 \mathrm{ml}), 1 \mathrm{M}$ Tris pH $7.5(500 \mu \mathrm{l})$ $\mathrm{dH} 20(43.5 \mathrm{ml})]$ and put at $95^{\circ} \mathrm{C}$ for 5 minutes. Protein concentration was assessed in a 96 well format by BCA Protein assay Kit (PIERCE Catalog number: 23225). $30 \mu \mathrm{g}$ of protein extract was loaded onto $10 \%$ Tris-Glycine SDSPAGE gels and run at 100 Volts for 90 minutes. The gels were transferred onto PVDF membrane and incubated overnight at $4^{\circ} \mathrm{C}$ with primary antibody,1:1000 rabbit Anti-MRE11 Antibody (NOVUS Biologicals Catalog number: NB100-276 diluted in 5\% non fat dry milk/TBS-T. The secondary antibody used was a 1:5000 dilution of ECL Anti-rabbit IgG Horseradish Peroxidase-Linked Species specific $F(a b$ ')2 Fragment (donkey) (Amersham Catalog number: NA9340). Membranes were incubated for 1 hour in secondary antibody and developed using Pierce Supersignal West Dura Extended Duration Substrate (Catalog number: 34705).

\section{Proliferation assays}

All of the cell lines were obtained from ATCC (Manassas, VA). MSI- DLD-1, HCT-8, HCT-15, HCT116, LOVO, LS174T, LS180, LS411N, RKO, RKOE6, SNUC2A, SW48. MSS- COLO205, HT29, NCI-H-508, SK-CO-1, SW403, SW480, SW620, SW837, SW948, SW1116, SW1417, SW1463, T84. To carry out 7 day monotherapy proliferation assays with the cell lines, 500-32,000 cells (cell linedependent) were seeded in 96-well clear tissue culture plates $(190 \mu \mathrm{l} /$ well $)$ in an appropriate tissue culture medium supplemented with FBS. The plate was incubated for 4 hours at $37^{\circ} \mathrm{C}$, and niraparib was added in a 9 point titration, 3-fold dilutions starting at $10 \mu \mathrm{M}$ for niraparib and starting at either $10 \mu \mathrm{M}, 1 \mu \mathrm{M}$, or $100 \mathrm{nM}$ for SN-38 (in 9 point titration, 3-fold dilutions). The cells were then incubated for 7 days at $37^{\circ} \mathrm{C}, 5 \% \mathrm{CO}_{2}$ (except cell lines 
grown in $\mathrm{L}-15$ medium which were grown in at $37^{\circ} \mathrm{C}$, $0 \% \mathrm{CO}_{2}, 100 \%$ air) and the cell viability was assessed by WST-1 assay (Roche) as described by the Manufacturer. To carry out 7 day combination proliferation assays with the cell lines, 500-32,000 cells (cell linedependent) were seeded in 96-well clear tissue culture plates $(180 \mu \mathrm{l} /$ well $)$ in an appropriate tissue culture medium supplemented with FBS. The plate was incubated for 4 hours at $37^{\circ} \mathrm{C}$, and niraparib was added at $125 \mathrm{nM}, 250 \mathrm{nM}$, or $1 \mu \mathrm{M}$ and SN-38 was added in an 8 point titration, 3 -fold dilutions, starting at $100 \mathrm{nM}$. The cells were then incubated for 7 days at $37^{\circ} \mathrm{C}, 5 \%$ $\mathrm{CO}_{2}$ (except for the cell lines grown in L-15 medium which were grown in at $37^{\circ} \mathrm{C}, 0 \% \mathrm{CO}_{2}, 100 \%$ air) and the cell viability was assessed by WST-1 assay (Roche) as described by the Manufacturer. The number of living cells was determined by reading the plate at $450 \mathrm{~nm}$ on a spectrophotometer. The signal produced is directly proportional to the cell number as the cells convert tetrazolium salt due into a formazan end product. Each experiment was run in duplicate. Cell growth was expressed as the percentage growth with respect to vehicle treated cells. The concentration required to inhibit cell growth by $50 \%\left(\mathrm{EC}_{50}\right)$ was determined using the four-parameter fit in SoftMax Pro 5.2. The Wilcoxon rank sum test was performed to determine statistical significance. Excess Volume HSA combination index was calculated using a MATLAB algorithm as described previously [26].

\section{In vivo xenograft studies}

6 week old CD1 nu/nu mice were injected subcutaneously with either $5 \times 10^{6}$ HT29 cells in $50 \%$ matrigel or $5 \mathrm{X} 10^{6}$ HCT116 cells in 50\% matrigel. When the average tumor size reached to $150 \mathrm{~mm}^{3}$ for HT29 and HCT116, mice were randomized to form homogeneous groups, and treatment started. Tumor measurements were recorded bi-weekly throughout the course of each study. Animals were dosed orally (p.o.) with 50,25 , or $10 \mathrm{mg} / \mathrm{kg}$ (mpk) niraparib $(5 \mathrm{ml} / \mathrm{kg}$ in $0.5 \% \mathrm{w} / \mathrm{v}$ methylcellulose) each day for 3, 5, or 7 days (according to individual study design) alone or in combination with $100 \mathrm{mpk}$ irinotecan $(10 \mathrm{ml} / \mathrm{kg})$ dosed intraperitoneally (ip.), once per week (qwk.), on day 3 for 4 weeks depending on treatment group. For tumor relapse studies, animals were treated for 4 weeks as described above, and then treatments were withdrawn and tumor relapse was monitored until the average tumor volume for each group reached $1000 \mathrm{~mm}^{3}$. Each animal study was conducted with 7-10 mice per individual treatment group. All animal studies were conducted in a specific pathogen-free environment in accordance with the internal Institutional Animal Care and Use Committee (IACUC) and other relevant standards.

\section{Results}

BAT-25 is a poly $(\mathbf{T})$ tract intragenic to the c-kit protooncogene assigned to $4 \mathrm{q} 12$ and BAT-26 is a poly (A) tract located in the $5^{\text {th }}$ intron of the hMSH2 gene. The BAT-25 and BAT-26 mononucleotide repeats are reported to be quasi-monomorphic meaning that there is not a significant size variation either between the alleles of one individual or between alleles of different individuals. This property permits the easy identification of MSI status without the use of a normal tissue control or on cell lines. MSI/MSS status across a CRC cell line panel was determined via PCR fragment analysis of the BAT-25 and BAT-26 mononucleotide repeats. MSS cell lines had longer and more uniform BAT-25 and BAT-26 alleles than MSI lines on average (Figure 1A and B). This data confirmed that the cell lines used in our cell line panel were MSI or MSS, as previously reported [20-25].

Cells with MSI are hypothesized to be HR-deficient due to reduced expression of Mre11 and subsequently the reduced expression of the Mre11-Nbs1-Rad50 complex. This reduction in expression has been shown to result from a mutation in the poly(T) 11 repeat within the human MRE11 intron 4 [6]. Western analysis confirmed reduced Mre11 protein levels in MSI as compared to MSS cell lines (Figure 1C), consistent with previous reports [6,7,10,27].

Enhanced sensitivity to PARP inhibition was postulated for MSI CRC cell lines due to the reduction in Mre11 protein expression. To test this hypothesis, the cell line panel was expanded (12 MSI and 13 MSS) and 7 -day proliferation assays were performed with niraparib. Although MSI cell lines on average did have lower 7 day proliferation EC50 values (Ave $=1823 \mathrm{nM}$ ) than MSS cell lines $($ Ave $=6859 \mathrm{nM})$, this difference was not statistically significant ( $\mathrm{p}=0.15$ Wilcoxon Rank Sum Test; Figure 2A and Table 1), in contrast to what has been previously reported [28,29]. A semi-quantitative assessment of the amount of Mre11 expression relative to GAPDH expression was also performed, and there was no correlation between the level of Mre11 expression and sensitivity to niraparib (data not shown).

Proliferation assays were also performed on the panel of cell lines with $\mathrm{SN}-38$. In agreement with previous reports, MSI CRC cell lines were significantly more sensitive to SN-38 monotherapy than MSS cells ( $p=0.02$ Wilcoxon Rank Sum Test; Figure 2B and Table 1) [18]. All of the MSI cell lines were sensitive to $\mathrm{SN}-38$ with EC50s less than $5 \mathrm{nM}$, and although on average the MSS cell lines were less sensitive, 6/13 cell lines did have EC50s less than $5 \mathrm{nM}$.

PARP inhibitors have been shown to potentiate the effects of Top 1 inhibitors both preclinically and clinically [12-17]. In order to test if niraparib potentiates irinotecan in MSI and MSS CRC cell lines, in vitro combination 

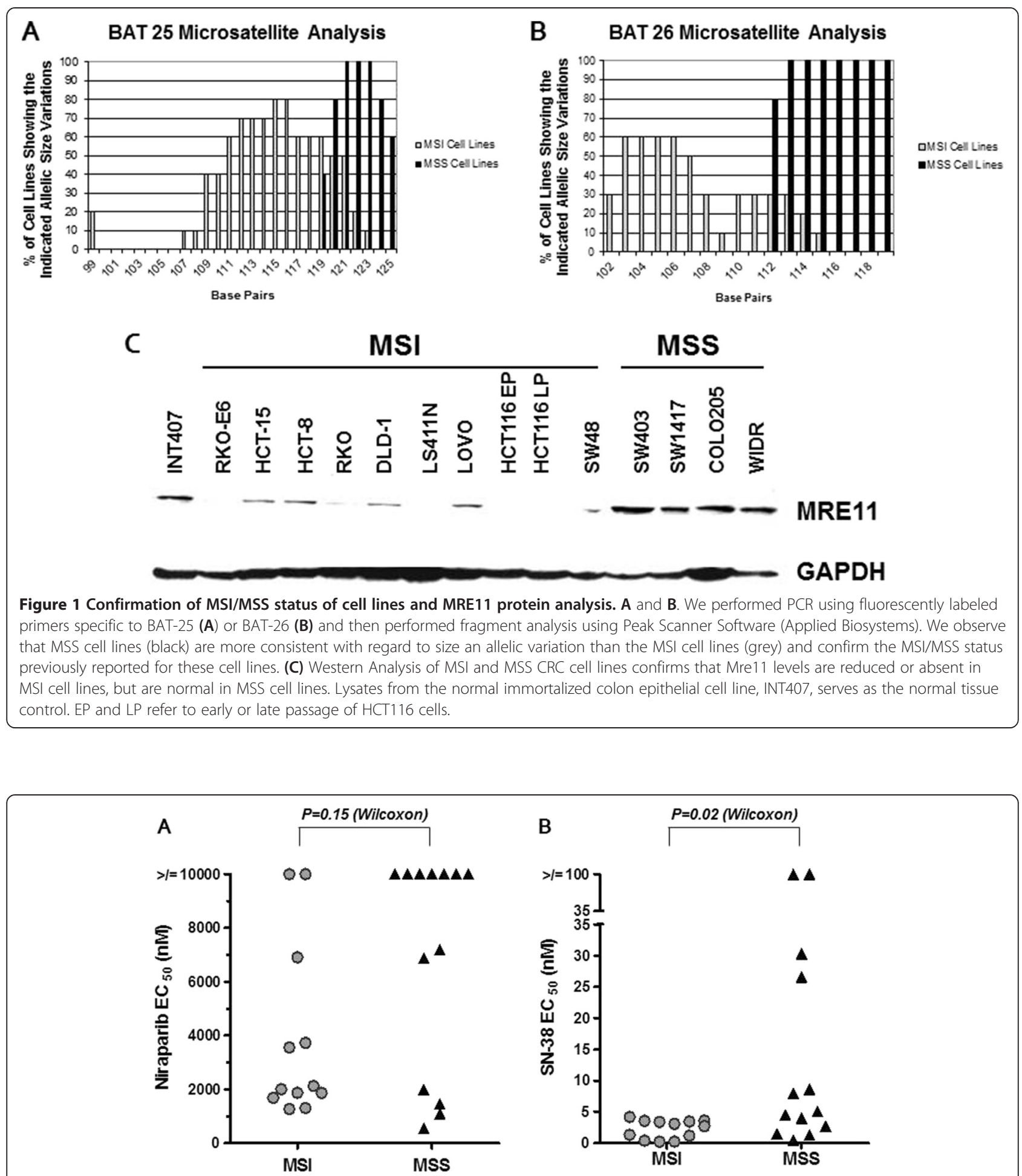

Figure 2 MSI cell lines do not respond better to niraparib monotherapy than MSS cell lines, but do respond better to SN-38 than MSS lines. A. MSI cell lines are not significantly more sensitive to niraparib than MSS cell lines. Niraparib EC50 values from 7 day proliferation assays in $12 \mathrm{MSI}$ and 13 MSS CRC cancer cell lines. Each symbol indicates the EC50 of a single cell line and results are representative of 2 independent experiments. Cell lines that did not respond to niraparib within the tested dose range (10000 nM and below) are shown at $10000 \mathrm{nM}$. B. MSI cell lines are more sensitive to SN-38 treatment than MSS cell lines. Niraparib EC50 values from 7 day proliferation assays in 12 MSI and 13 MSS CRC cancer cell lines. Each symbol indicates the EC50 of a single cell line and results are representative of 2 independent experiments. Cell lines that did not respond to niraparib within the tested dose range (100 nM and below) are shown at $100 \mathrm{nM}$. The Wilcoxon rank sum test was performed to determine statistical significance. 
Table 1 Niraparib potentiates the effects of SN-38 in MSI and MSS cell lines

\begin{tabular}{|c|c|c|c|c|c|c|}
\hline & \multirow[b]{3}{*}{ Cell line } & \multicolumn{2}{|c|}{7 day EC50 (nM) } & \multicolumn{3}{|c|}{ Combination EC50 (shift of SN-38 EC50 in presence of Niraparib) } \\
\hline & & \multirow[b]{2}{*}{ Niraparib } & \multirow[b]{2}{*}{ SN-38 } & \multicolumn{3}{|c|}{ Niraparib } \\
\hline & & & & $125 \mathrm{nM}$ & $250 \mathrm{nM}$ & $1000 \mathrm{nM}$ \\
\hline \multirow[t]{12}{*}{ MSI } & DLD1 & 3560 & 3.63 & 0.992 & 1.67 & 1.68 \\
\hline & HCT8 & 2130 & 3.60 & 2.07 & 1.54 & 1.35 \\
\hline & HCT15 & 1685 & 3.77 & 3.47 & 3.44 & 1.73 \\
\hline & HCT116 & 6915 & 0.180 & 0.125 & 0.155 & 0.0991 \\
\hline & LOVO & 2010 & 3.43 & 2.3 & 3.53 & 2.4 \\
\hline & LS174T & 1310 & 0.331 & 0.257 & 0.208 & 0.231 \\
\hline & LS180 & 3730 & 0.474 & 0.399 & 0.395 & 0.389 \\
\hline & LS411N & 1875 & 3.57 & 1.21 & 1.11 & 0.729 \\
\hline & RKO & 1870 & 0.958 & 0.593 & 0.778 & 0.184 \\
\hline & RKOE6 & 1270 & 0.730 & 0.426 & 0.422 & 0.464 \\
\hline & SNUC2A & $>10000$ & 3.23 & 3.04 & 2.72 & 2.52 \\
\hline & SW48 & $>10000$ & 1.79 & 0.886 & 0.762 & 0.598 \\
\hline \multirow[t]{13}{*}{ MSS } & COLO205 & 1990 & 1.57 & 0.572 & 0.475 & 0.531 \\
\hline & HT29 & 6880 & 4.29 & 3.07 & 3.64 & 1.75 \\
\hline & $\mathrm{NCl}-\mathrm{H}-508$ & $>10000$ & 3.63 & 1.70 & 3.31 & 1.41 \\
\hline & SK-CO-1 & 1090 & 0.416 & 0.178 & 0.334 & 0.143 \\
\hline & SW403 & $>10000$ & 3.39 & 1.14 & 1.87 & 0.387 \\
\hline & SW480 & $>10000$ & 29.0 & 11.9 & 11.6 & 8.7 \\
\hline & SW620 & 552 & 5.91 & 3.62 & UR & UR \\
\hline & SW837 & $>10000$ & UR & UR & UR & UR \\
\hline & SW948 & 7195 & 6.67 & 3.85 & 4.47 & 2.80 \\
\hline & SW1116 & $>10000$ & UR & UR & UR & UR \\
\hline & SW1417 & $>10000$ & 27.7 & 29.2 & 28.3 & 31.0 \\
\hline & SW1463 & $>10000$ & 26.4 & 22.2 & UR & UR \\
\hline & T84 & 1460 & 1.01 & 0.491 & 0.352 & 0.210 \\
\hline
\end{tabular}

7 day EC50 data for niraparib alone, SN-38 alone, and the combination EC50 at 125, 250 and 1000 nM of niraparib in a panel of 25 MSI and MSS CRC cell lines . The niraparib EC50 values are the average of $n=2$ from previous experiments. The SN-38 EC50 values reported are for $n=1$ for the experiment done on that day. Values listed under 125, 250, and 1000nM niraparib are the EC50 values for SN-38 in the presence of 125, 250 or $1000 \mathrm{nM}$ niraparib (combination EC50). EC50 data was calculated using the inflection point of the curve in a 4 parameter fit in SoftMax Pro 5.2. UR indicates that the data is unreportable due to an inadequate curve.

studies with niraparib $(125 \mathrm{~nm}, 250 \mathrm{nM}$, and $1000 \mathrm{nM})$ and $\mathrm{SN}-38$ (8 point dose response) were performed. As expected, we observed a shift of the SN-38 EC50 an average of 2-fold lower when niraparib was tested in combination with $\mathrm{SN}-38$ as compared to when SN-38 was tested alone in both MSI and MSS CRC cell lines (Table 1). This data demonstrates that if a cell line responds to SN-38 monotherapy in vitro, niraparib potentiates that effect, regardless of MSI/MSS status. In addition, when Highest Single Agent (HSA) combinatorial analysis was applied to this data, we observe that combination of niraparib with SN-38 results in additive to synergistic inhibition of cell proliferation regardless of MSI/MSS status in vitro (Figure 3).

In order to extend these observations, xenograft studies employing MSI (HCT116) or MSS (HT29) models were performed. HCT116 (MSI) or HT29 (MSS) xenograft tumor-bearing mice were dosed with niraparib at 10,25 , or $50 \mathrm{mpk}$ (oral, daily) for 5 days per cycle (4 cycles) and efficacy at these doses was compared to irinotecan monotherapy (100 mpk, ip., qwk; 4 cycles). In both models, irinotecan monotherapy was more efficacious than niraparib monotherapy at the niraparib doses tested (Figure 4). In HCT116, the average tumor volume at day 28 for the irinotecan treated group was $225 \mathrm{~mm}^{3}$ and the tumor volumes for the niraparib single agent treated groups were 605,734 and $739 \mathrm{~mm}^{3}$ for $10 \mathrm{mpk}$, $25 \mathrm{mpk}$ and $50 \mathrm{mpk}$ niraparib treated groups, respectively. When compared to vehicle control, only the average tumor volume of the irinotecan treated group was statistically different than the average of vehicle control ( $\mathrm{p}=0.0002$ one-tailed homoscedastic student's $\mathrm{T}$ test). In 


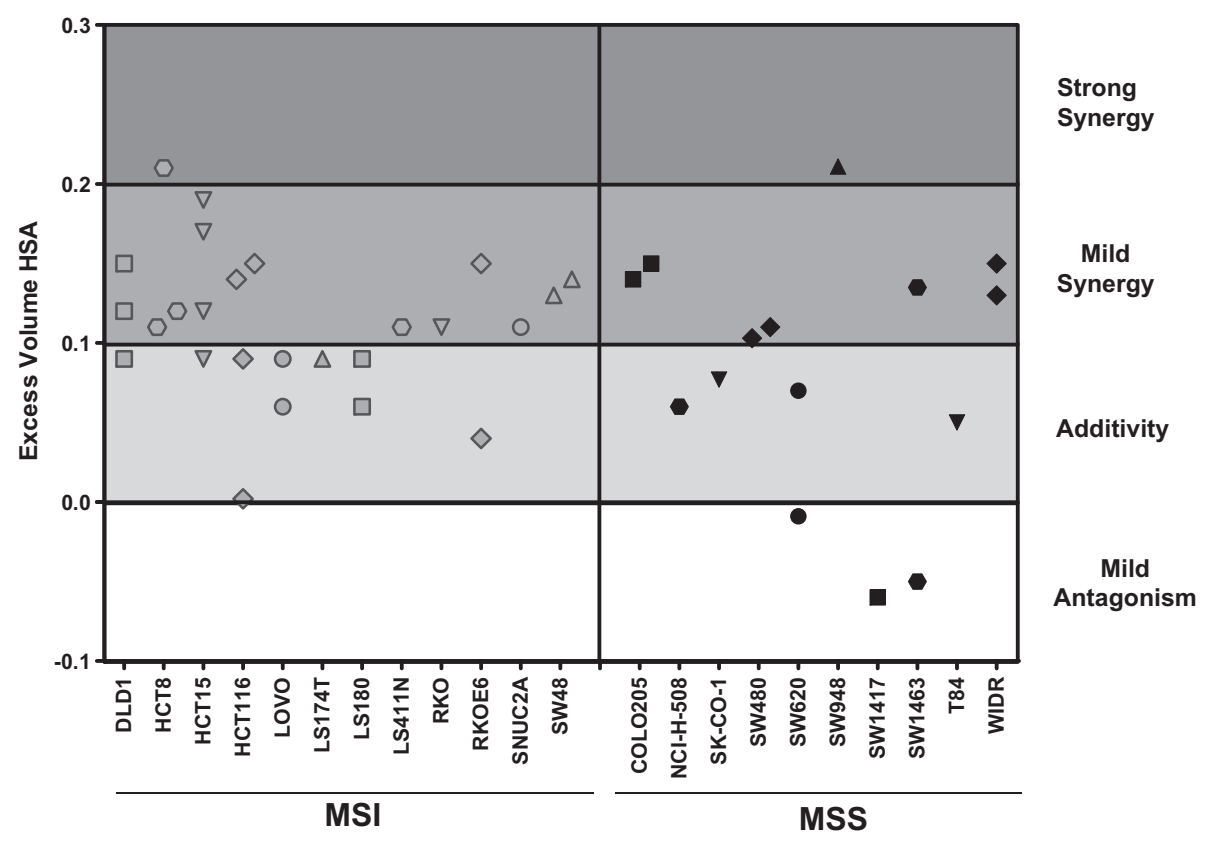

Figure 3 Niraparib potentiates the effects of SN-38 in MSI and MSS cell lines. Assessment of synergy with niraparib and SN-38 by excess volume HSA. Each symbol indicates a single experiment with the indicated cell lines. Data was generated from 7 day proliferation assay and excess volume HSA was calculated using a MATLAB algorithm. HSA values from 0.2-0.3 indicate strong synergy, 0.1-0.2 indicated mild synergy, 0.0-0.1 indicate additivity, and negative numbers indicate mild antagonism. Data points are from 1-3 experiments and are only shown if the $p$ value associated with the excess volume HSA is equal to or less than 0.05 .

HT29, the average tumor volume for the irinotecan treated group at day 24 was $542 \mathrm{~mm}^{3}$ whereas the tumor volumes for the niraparib treated groups were 934, 802 and $768 \mathrm{~mm}^{3}$, for the $10 \mathrm{mpk}, 25 \mathrm{mpk}$ and 50 mpk, niraparib treated groups, respectively. (Tumor volumes at day 24 were recorded for HT29 as compared to day 28 for HCT116 due to the day when vehicle control treated groups reached the maximum tumor volume of $1000 \mathrm{~mm}^{3}$.) In the HT29 model, none of the single agent groups (including irinotecan) had average tumor volumes that were statistically different as compared to vehicle control (irinotecan versus vehicle control $\mathrm{p}$ value $=0.06$ one-tailed homoscedastic student's $\mathrm{T}$ test). Niraparib was dosed no higher than $50 \mathrm{mpk}$ in these studies because higher concentrations of niraparib were not tolerated in combination with irinotecan dosed at $100 \mathrm{mpk}$, qwk (data not shown). The maximum tolerated dose for niraparib as a single agent in mice is $100 \mathrm{mpk}$ daily (data not shown). Doses of niraparib at $50 \mathrm{mpk}$ (daily) gives approximately the same $\mathrm{C}$ min values as $40 \mathrm{mg} /$ day in humans, the lowest dose that demonstrated clinical benefit (stable disease) in humans [30].

In the same studies, mice were also treated with niraparib (at 10, 25, and $50 \mathrm{mpk}$ ) in combination with irinotecan (100 mpk; qwk) to determine if niraparib could enhance irinotecan efficacy, and to determine if the combination would be tolerated. Niraparib significantly enhanced irinotecan efficacy at the 25 and 50 mpk combination dosing regimens in the HCT116 model, but not in the $10 \mathrm{mpk}$ combination dosing regimen. The average tumor volume at day 28 in the Irinotecan group was $225 \mathrm{~mm}^{3}$, in the $50 \mathrm{mpk}$ combination group was $120 \mathrm{~mm}^{3}$, the $25 \mathrm{mpk}$ combination group was $101 \mathrm{~mm}^{3}$ and in the $10 \mathrm{mpk}$ combination group was $166 \mathrm{~mm}^{3}$. Significance was determined using a one-tailed homoscedastic $\mathrm{T}$ test and $\mathrm{p}=0.01,0.02$ and 0.06 for the 50 mpk, 25 mpk and $10 \mathrm{mpk}$ combination groups as compared to irinotecan alone at day 28. In the HT29 model, the average tumor volumes for the combination groups were smaller at 24 days as compared to irinotecan, but these values were not statistically significant. The average tumor volume at day 24 in the Irinotecan group was $542 \mathrm{~mm}^{3}$, in the $50 \mathrm{mpk}$ combination group was $363 \mathrm{~mm}^{3}$, the $25 \mathrm{mpk}$ combination group was $438 \mathrm{~mm}^{3}$ and in the $10 \mathrm{mpk}$ combination group was $448 \mathrm{~mm}^{3}$. Significance was again determined using a one-tailed homoscedastic $\mathrm{T}$ test and $\mathrm{p}=0.09,0.15$ and 0.18 for the 50 mpk, $25 \mathrm{mpk}$ and $10 \mathrm{mpk}$ combination groups as compared to irinotecan alone at day $24.15 \%$ or less body weight loss was observed throughout the duration of the studies (Figures 4C, 4D, 5C and 5D).

In addition to assessing tumor growth inhibition during the 4 weeks with niraparib and irinotecan combination treatment, we also investigated tumor growth 

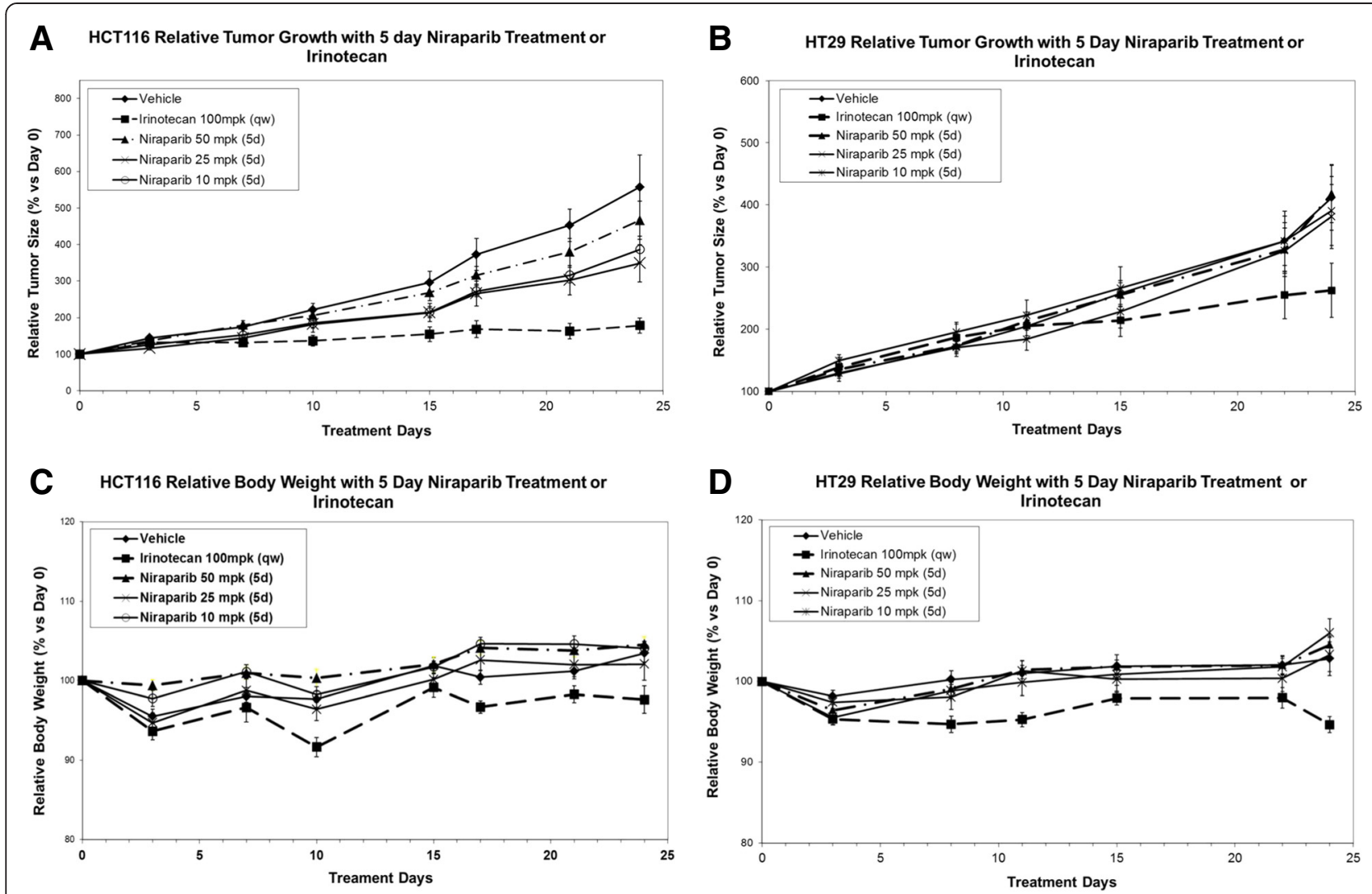

Figure 4 Niraparib monotherapy is not as efficacious as irinotecan monotherapy in the HCT116 (MSI) or HT29 (MSS) xenograft models.

A. and B. In vivo efficacy of niraparib monotherapy on the HCT116 (MSI) and HT29 (MSS) xenograft models. Niraparib was dosed at 50, 25, or 10 mpk p.o. q.d., for 5 days a week (2 days off) for 4 weeks. Relative tumor size (\% versus Day 0 ) is shown with SEM in error bars. C. and D. The 5 day dosing regimen was well tolerated with less than an average of $10 \%$ body weight loss in all doses tested. Relative body weight (\% versus Day 0 ) is shown. For each group, $n=7-10$.

delay and relapse in the same studies after the withdrawal of treatments. The HCT116 and HT29 tumors treated with irinotecan alone relapsed sooner than tumors treated with niraparib in combination with irinotecan at all 3 niraparib doses tested (Figure 6). In the HCT116 study, the $50 \mathrm{mpk}$ niraparib + irinotecan combination treatment group reached the $1000 \mathrm{~mm}^{3}$ endpoint at day 99, whereas the irinotecan alone treatment group reached the $1000 \mathrm{~mm}^{3}$ endpoint at day 70 , demonstrating a 29-day tumor growth delay. Additionally, the average tumor volume for the 50,25 , and 10 mpk niraparib + irinotecan combination groups in the HCT116 model were significantly different from the irinotecan single agent group at day 70 when the average tumor size in the irinotecan single agent group reached the end-point of $1000 \mathrm{~mm}^{3} .(\mathrm{P}=.02, .02$, and .03 , respectively. $\mathrm{P}$ values generated using a homoscedastic Student's t-test.) (Figure 6A). Likewise, in the HT29 study, the 50 mpk niraparib + irinotecan combination treatment group reached the $1000 \mathrm{~mm}^{3}$ endpoint at day 65 , whereas the irinotecan only treatment group reached the end-point at day 49 , demonstrating a 16-day tumor growth delay. The average tumor volume for the 50, 25, and $10 \mathrm{mpk}$ niraparib + irinotecan combination groups in the HT29 model were significantly different from the irinotecan single agent group at day 49 when the average tumor size in the irinotecan single agent group reached the end-point of $1000 \mathrm{~mm}^{3}$. (P-values of $.05, .03$, and .03 , respectively. $\mathrm{P}$ value generated using a homoscedastic Student's t-test) (Figure 6B). Collectively, our in vitro and in vivo data demonstrate that combination of niraparib with irinotecan is efficacious in both MSI and MSS settings.

\section{Discussion}

To determine if MSI is associated with increased sensitivity to the PARP inhibitor niraparib and to determine if niraparib potentiates the anti-proliferative effects of irinotecan, the efficacy of niraparib and irinotecan, both alone and in combination, was assessed in multiple MSI and MSS CRC models in vitro and in vivo. The studies detailed in this paper demonstrate that CRC MSI cell lines are not more sensitive than CRC MSS cell lines to niraparib, and that combination of niraparib with 

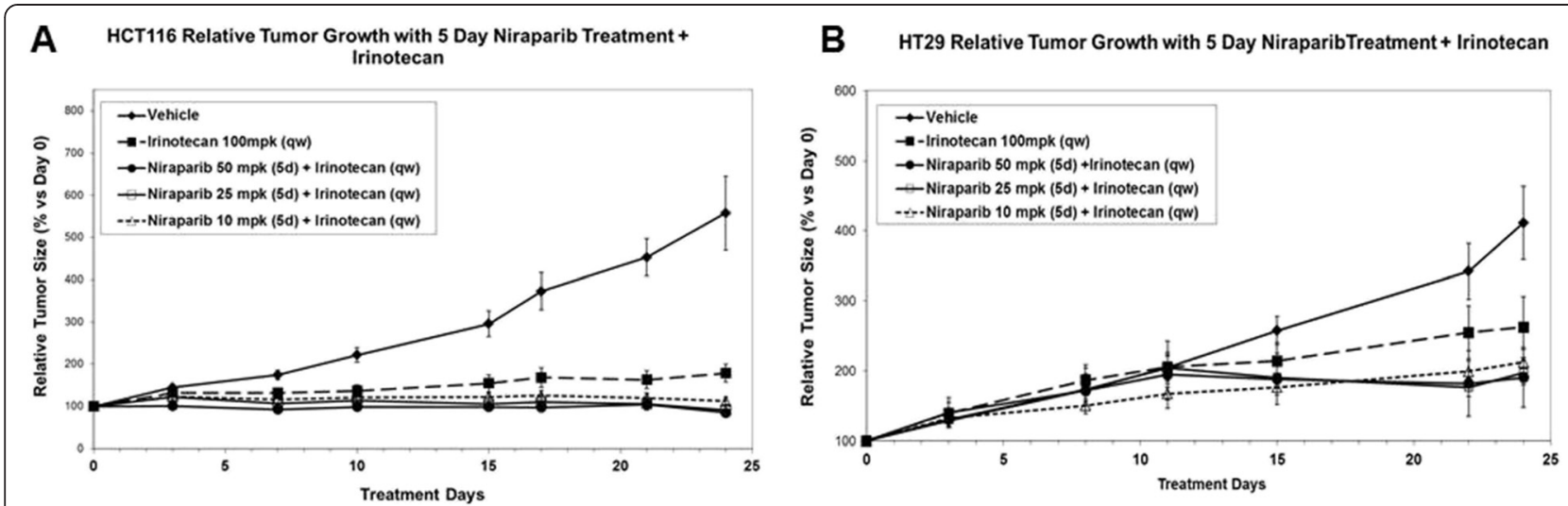

\section{HCT116 Relative Body Weight with 5 Day NiraparibTreatment +}

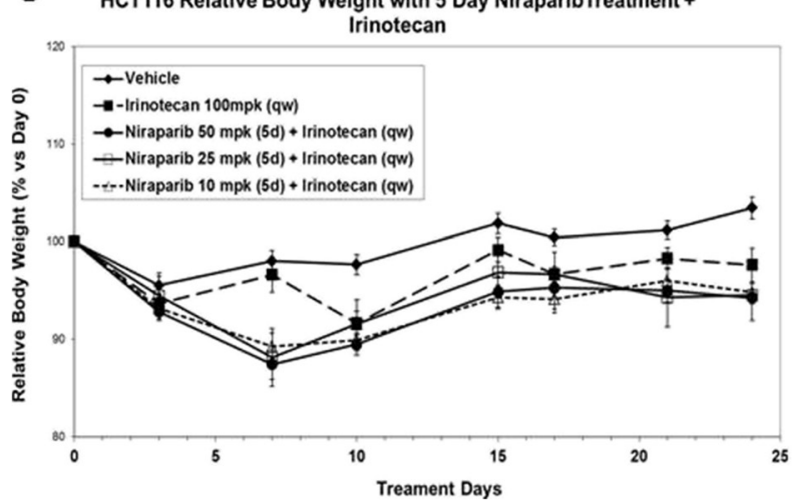

D HT29 Relative Body Weight with 5 Day Niraparib Treatment + Irinotecan

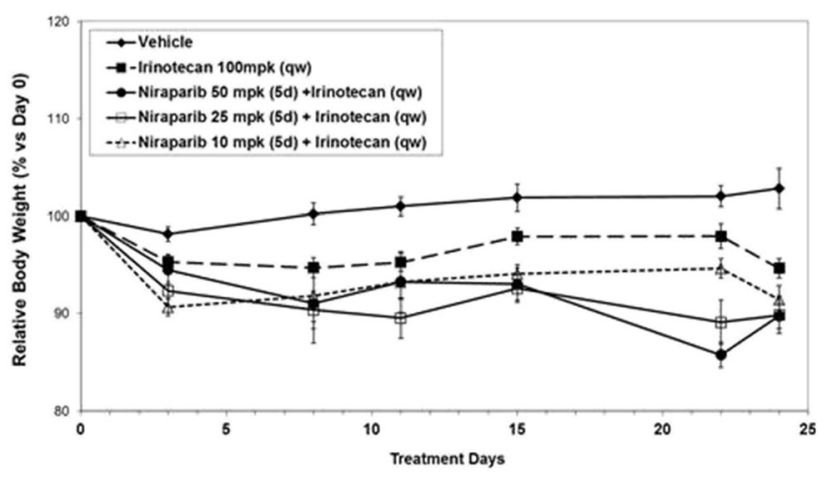

Figure 5 Niraparib in combination with irinotecan is more efficacious than irinotecan alone in the HCT16 (MSI) CRC and HT29 (MSS) models. (Data from this figure is from the same experiment as in Figure 4, but has been separated for ease of viewing.) In vivo efficacy of niraparib in combination with irinotecan in the A. HCT116 (MSI) and B. HT29 (MSS) xenograft models. Niraparib was dosed at 50, 25, or 10 mpk p. o. q.d., for 5 days a week ( 2 days off) and irinotecan was administered at 100 mpk i.p., on the 3 rd day of every week for 4 weeks. Relative tumor size (\% versus Day 0) is shown with SEM in error bars. $\mathbf{C}$. and $\mathbf{D}$. The 5-day dosing regimen was tolerated with $15 \%$ or less body weight loss in all combinations tested. Relative body weight (\% versus Day 0 ) is shown. For each group, $n=7-10$.

irinotecan enhances the efficacy of irinotecan in both MSI and MSS CRC cell lines in vitro and in vivo.

We have demonstrated that MSI CRC cell lines have reduced levels of Mre11, a protein involved in the repair of DSBs, as compared to MSS cell lines, but that they are not significantly more sensitive to niraparib monotherapy than MSS cell lines (Figures 1 and 2A). Even the cell lines that had little or no detectable levels of Mre11 (RKO, LS411N, HCT116; Figure 1) did not respond to niraparib monotherapy in the EC50 ranges that BRCA deficient cell lines do (EC50s $\geq 1000 \mathrm{nM}$ for MSI CRC cell lines and EC50s $\leq 100 \mathrm{nM}$ for BRCA1/2 mutant cell lines using the same assay conditions) (Figure $2 \mathrm{~A}$ and Table 1; [3]). This data demonstrates that reduction of Mre11 levels to the degree that they are reduced in the context of MSI, is not sufficient to induce sensitivity to PARP inhibition. The notion that Mre11 deficiency is fundamentally different from BRCA $1 / 2$ deficiency is supported by the observation that germline inactivation of Mre11 does not result in a cancer predisposition syndrome, whereas inactivation of BRCA $1 / 2$ does.
Our results differ from those published for the PARP inhibitor ABT-888 by Vilar et al., in 2011 and the PARP inhibitor BMN673 by Gaymes et al., in 2013 [28,29]. Vilar et al. reported that Mre11 deficiency increases sensitivity to PARP inhibition in MSI CRC. Discrepancies in our results are likely due to the size of the cell line panels that were used. The panel used in our study was larger than what Vilar et al., used. All of the MSI cell lines that were assayed in the Vilar manuscript were assayed in our panel with an additional 4 MSI lines. In addition, 7 out of the 9 of the MSS cell lines that were assayed in their panel were assayed in our panel with an additional 6 MSS lines. When statistical analysis is performed on our 7 day niraparib EC50 data using only the cell lines that were used in the Vilar study, we demonstrate that MSI cell lines are significantly more sensitive to niraparib than MSS cell lines $(p=0.02)$. This data highlights the need to include large numbers of cell lines to evaluate biomarker hypotheses. Additionally, Vilar et al., describe the differences between MSI and MSS cell lines using $10 \mu \mathrm{M}$ (and $50 \mu \mathrm{M}$ ) of ABT-888, which 
A HCT116 Relative Tumor Growth with 5 day Niraparib Treatment +

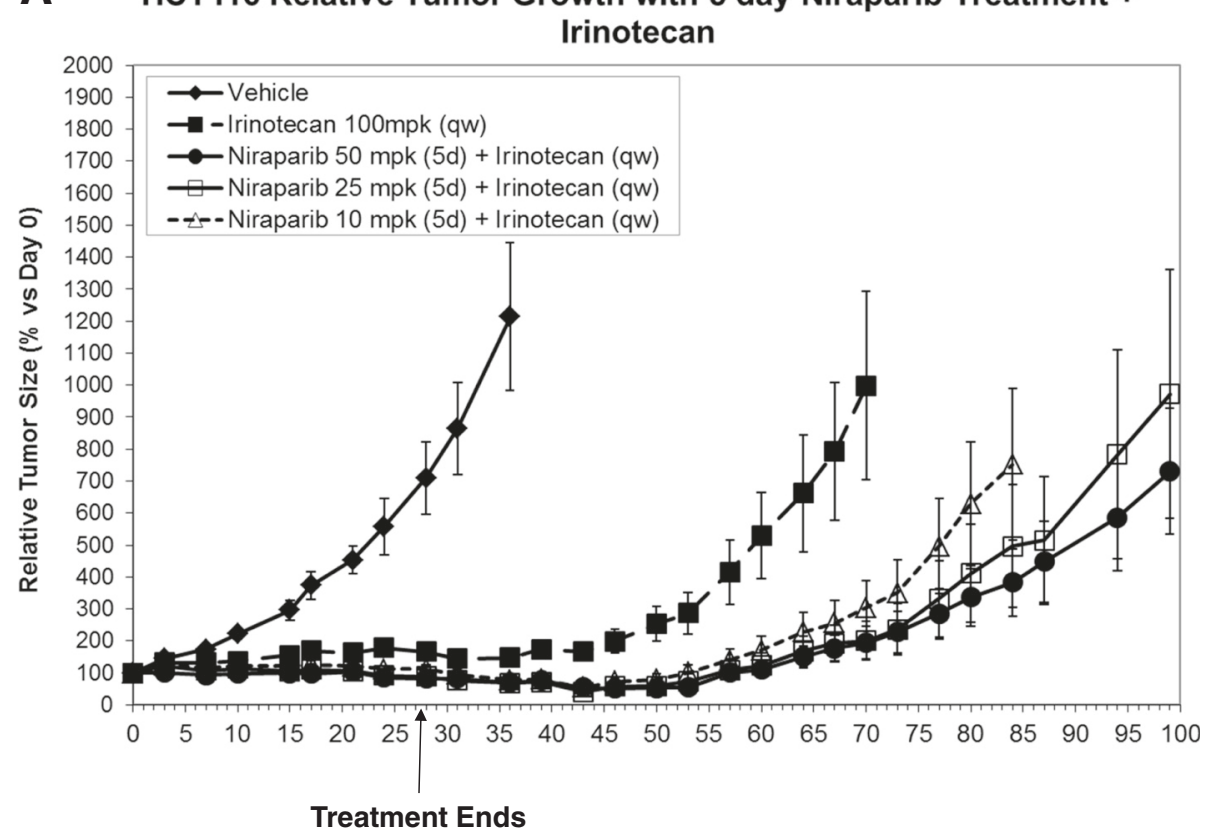

Treatment Days

B HT29 Relative Tumor Size with 5 Day Niraparib Treatment + Irinotecan

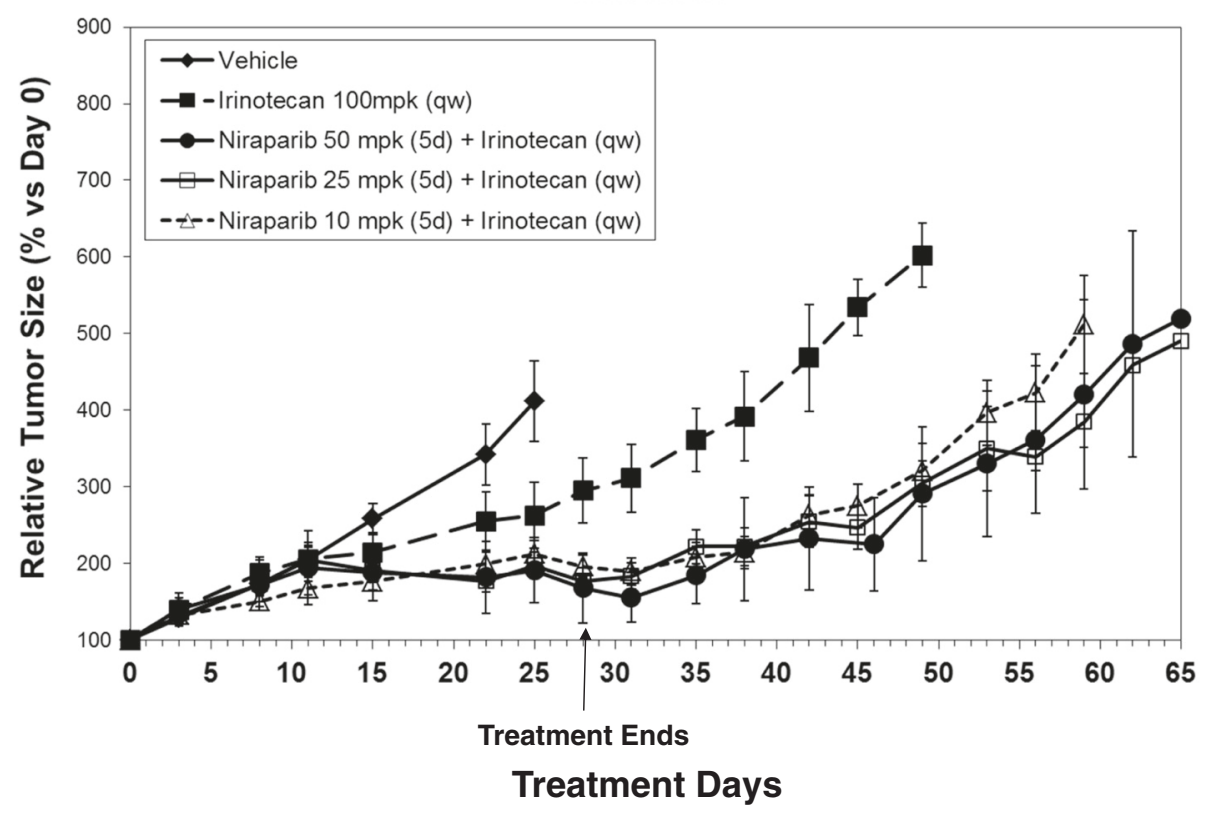

Figure 6 (See legend on next page.) 


\section{(See figure on previous page.)}

Figure 6 Tumor growth delay is extended signficantly in niraparib + irinotecan combination treatment groups as compared to treatment with irinotecan alone. (Data from this figure is from the same experiment as in Figures 4 and 5 , but has been separated for ease of viewing). Tumor growth delay and relapse after the withdrawal of niraparib and irinotecan treatment in the (A) HCT116 (MSI) and (B) HT29 xenograft models. Niraparib was dosed at 50, 25, or 10 mpk p.o. q.d., for 5 days and irinotecan was administered at 100 mpk i.p. on the 3 rd day of every week for 4 weeks. After the $4^{\text {th }}$ week of dosing of both drugs, treatment was stopped and tumor growth was monitored bi-weekly until the average tumor volume for each group reached to an end-point of $1000 \mathrm{~mm}^{3}$. Relative tumor size (\% versus Day 0 ) is shown with SEM in error bars. For each group, $n=7-10$. (A) The average tumor volume for the 50, 25, and 10 mpk niraparib + irinotecan combination groups in the HCT116 model were significantly different from the irinotecan single agent group at day 70 when the average tumor size in the irinotecan single agent group reached the end-point of $1000 \mathrm{~mm}^{3}$ with P-values of .02, .02, and .03, respectively. (P value generated using a homoscedastic Student's t-test.) (B) The average tumor volume for the 50, 25, and 10 mpk niraparib + irinotecan combination groups in the HT29 model were significantly different from the irinotecan single agent group at day 49 when the average tumor size in the irinotecan single agent group reached the end-point of $1000 \mathrm{~mm}^{3}$ with P-values of .05, .03, and .03, respectively. (P value generated using a homoscedastic Student's t-test).

is more than 10 times the EC50 reported for ABT-888 in the BRCA mutant context [31]. Notably, the concentrations of compound used in those studies is unlikely a therapeutically relevant dose [32].

Gaymes et al., reported that MSI induced mutations in DNA repair genes confer hypersensitivity to the PARP inhibitor BMN673 in myeloid malignancies [29]. These authors were studying cell lines of myeloid lineage and we did not assay any of the same cell lines in our studies. Discrepancies in our results could stem from different cell line panels, the effects of MSI in other tissue types, or a difference in assay platform.

We have also confirmed previous reports that MSI CRC cell lines are more sensitive to irinotecan (SN-38) monotherapy than MSS CRC cell lines in a large cell line panel. (Figure 2B and Table 1) [18]. The average 7 day EC50 for SN-38 in MSI cell was $2.1 \mathrm{nM}$ and for MSS cells was $10.0 \mathrm{nM}$. However, 6/13 MSS cell lines tested had EC50s less than 5nM (Figure 2B and Table 1). The data in Figure 2B. demonstrates that there are SN-38 sensitive and SN-38 insensitive subpopulations of MSS cell lines. Some of the MSS cell lines are quite sensitive to $\mathrm{SN}-38$ and we have demonstrated that if a cell line responds to SN-38 monotherapy then niraparib will potentiate that effect, in both MSI and MSS cell lines (Table 1). We have also demonstrated that combination of niraparib with $\mathrm{SN}-38$ results in additive to synergistic inhibition of cell proliferation in both MSI and MSS CRC cell lines in vitro (Figure 3 ). In addition, we demonstrate that while the doses of niraparib used in this study are not as efficacious as irinotecan in monotherapy, the combination of low doses of niraparib and irinotecan results in greater tumor growth inhibition in MSI and MSS tumor models in vivo (Figure 4.) In tumor growth delay and relapse studies, tumors treated with irinotecan alone relapsed earlier than tumors treated niraparib and irinotecan combination at all three niraparib doses tested $(50,25,10 \mathrm{mpk})$ in both MSI and MSS CRC xenograft models.

Our in vitro and in vivo preclinical results demonstrate that in CRC cell lines, MSI does not render cells more sensitive to niraparib, but that combination of niraparib with irinotecan enhances the efficacy of irinotecan in both MSI and MSS CRC cell lines. Our data suggests that both MSI and MSS patient populations will benefit from the combination of niraparib and irinotecan.

\section{Competing interests}

SW is an employee of Merck Research Laboratories. The authors declare that they have no competing interests.

\section{Authors' contributions}

SW contributed to study design and coordination, drafted the manuscript, constructed the figures, carried out the microsatellite repeat analysis, performed the Western analysis, and performed the in vitro proliferation assays (single agent and combination). AK and PA carried out in vivo studies. BD performed statistical analyses. RO contributed manuscript preparation. CE contributed to study design and coordination. CT contributed to study concept, design and coordination. All authors read and approved the final manuscript.

\section{Acknowledgements}

We thank Stuart Shumway, Leigh Zawel, and Keith Wilcoxen for critical reading of this manuscript.

\section{Author details}

${ }^{1}$ Department of Oncology, Merck Research Laboratories, 33 Avenue Louis Pasteur, Boston, MA 02115, USA. ²Department of In Vivo Pharmacology, Merck Research Laboratories, 33 Avenue Louis Pasteur, Boston, MA 02115, USA. ${ }^{3}$ Current address: Bayer HealthCare, 100 Bayer Road, Whippany, NJ 07891, USA. ${ }^{4}$ Current address: Institute for Applied Cancer Science, 1901 East Road, Unit 1956, Room 4SCR6.1009, Houston, TX 77005, USA.

Received: 27 November 2013 Accepted: 14 January 2015 Published online: 04 February 2015

\section{References}

1. Bryant HE, Schultz N, Thomas HD, Parker KM, Flower D, Lopez E, et al. Specific killing of BRCA2-deficient tumours with inhibitors of poly(ADP-ribose) polymerase. Nature. 2005;434:913-7.

2. Farmer H, McCabe N, Lord CJ, Tutt AN, Johnson DA, Richardson TB, et al. Targeting the DNA repair defect in BRCA mutant cells as a therapeutic strategy. Nature. 2005;434:917-21.

3. Jones P, Altamura S, Boueres J, Ferrigno F, Fonsi M, Giomini C, et al. Discovery of 2-\{4-[(3S)-piperidin-3-yl]phenyl\}-2H-indazole-7-carboxamide (MK-4827): a novel oral poly(ADP-ribose)polymerase (PARP) inhibitor efficacious in BRCA-1 and -2 mutant tumors. J Med Chem. 2009:52:7170-85.

4. McCabe N, Turner NC, Lord CJ, Kluzek K, Bialkowska A, Swift S, et al. Deficiency in the repair of DNA damage by homologous recombination and sensitivity to poly(ADP-ribose) polymerase inhibition. Cancer Res. 2006;66:8109-15.

5. Fong PC, Boss DS, Yap TA, Tutt A, Wu P, Mergui-Roelvink M, et al. Inhibition of poly(ADP-ribose) polymerase in tumors from BRCA mutation carriers. N Engl J Med. 2009;361:123-34 
6. Giannini G, Ristori E, Cerignoli F, Rinaldi C, Zani M, Viel A, et al. Human MRE11 is inactivated in mismatch repair-deficient cancers. EMBO Rep. 2002;3:248-54

7. Duval A, Hamelin R. Mutations at coding repeat sequences in mismatch repair-deficient human cancers: toward a new concept of target genes for instability. Cancer Res. 2002;62:2447-54.

8. Koh KH, Kang HJ, Li LS, Kim NG, You KT, Yang E, et al. Impaired nonhomologous end-joining in mismatch repair-deficient colon carcinomas. Lab Invest. 2005:85:1130-8.

9. Fallik D, Borrini F, Boige V, Viguier J, Jacob S, Miquel C, et al. Microsatellite instability is a predictive factor of the tumor response to irinotecan in patients with advanced colorectal cancer. Cancer Res. 2003;63:5738-44

10. Miquel C, Jacob S, Grandjouan S, Aime A, Viguier J, Sabourin JC, et al. Frequent alteration of DNA damage signalling and repair pathways in human colorectal cancers with microsatellite instability. Oncogene. 2007;26:5919-26.

11. Ratnam K, Low J. Current Development of Clinical Inhibitors of Poly (ADP-Ribose) Polymerase in Oncology. Clin Cancer Res. 2007;13:1383-8.

12. Ray CA, Hashimoto Y, Herrador R, Neelsen KJ, Fachinetti D, Bermejo R, et al Topoisomerase I poisoning results in PARP-mediated replication fork reversal. Nat Struct Mol Biol. 2012;19:417-23.

13. Delaney CA, Wang LZ, Kyle S, White AW, Calvert AH, Curtin NJ, et al. Potentiation of temozolomide and topotecan growth inhibition and cytotoxicity by novel poly(adenosine diphosphoribose) polymerase inhibitors in a panel of human tumor cell lines. Clin Cancer Res. 2000;6:2860-7.

14. Smith LM, Willmore E, Austin CA, Curtin NJ. The novel poly(ADP-Ribose) polymerase inhibitor, AG14361, sensitizes cells to topoisomerase I poisons by increasing the persistence of DNA strand breaks. Clin Cancer Res. 2005:11:8449-57.

15. Patel AG, Flatten KS, Schneider PA, Dai NT, McDonald JS, Poirier GG, et al. Enhanced killing of cancer cells by poly(ADP-ribose) polymerase inhibitors and topoisomerase I inhibitors reflects poisoning of both enzymes. J Biol Chem. 2012;287:4198-210.

16. Kummar S, Chen A, Ji J, Zhang Y, Reid JM, Ames M, et al. Phase I study of PARP inhibitor ABT-888 in combination with topotecan in adults with refractory solid tumors and lymphomas. Cancer Res. 2011;71:5626-34.

17. Tentori L, Leonetti C, Scarsella M, Muzi A, Mazzon E, Vergati M, et al. Inhibition of poly(ADP-ribose) polymerase prevents irinotecan-induced intestinal damage and enhances irinotecan/temozolomide efficacy against colon carcinoma. FASEB J. 2006;20:1709-11.

18. Vilar E, Scaltriti M, Balmana J, Saura C, Guzman M, Arribas J, et al. Microsatellite instability due to hMLH1 deficiency is associated with increased cytotoxicity to irinotecan in human colorectal cancer cell lines. $\mathrm{Br}$ J Cancer. 2008;99:1607-12.

19. Bertagnolli MM, Niedzwiecki D, Compton CC, Hahn HP, Hall M, Damas B, et al. Microsatellite instability predicts improved response to adjuvant therapy with irinotecan, fluorouracil, and leucovorin in stage III colon cancer: Cancer and Leukemia Group B Protocol 89803. J Clin Oncol. 2009:27:1814-21.

20. Zhou XP, Hoang JM, Cottu P, Thomas G, Hamelin R. Allelic profiles of mononucleotide repeat microsatellites in control individuals and in colorectal tumors with and without replication errors. Oncogene. 1997;15:1713-8.

21. Hoang JM, Cottu PH, Thuille B, Salmon RJ, Thomas G, Hamelin R. BAT-26, an indicator of the replication error phenotype in colorectal cancers and cell lines. Cancer Res. 1997:57:300-3.

22. Efstathiou JA, Liu D, Wheeler JM, Kim HC, Beck NE, Ilyas M, et al. Mutated epithelial cadherin is associated with increased tumorigenicity and loss of adhesion and of responsiveness to the motogenic trefoil factor 2 in colon carcinoma cells. Proc Natl Acad Sci U S A. 1999:96:2316-21.

23. Shibata D, Peinado MA, lonov Y, Malkhosyan S, Perucho M. Genomic instability in repeated sequences is an early somatic event in colorectal tumorigenesis that persists after transformation. Nat Genet. 1994;6:273-81.

24. Umar A, Boyer JC, Thomas DC, Nguyen DC, Risinger Jl, Boyd J, et al. Defective mismatch repair in extracts of colorectal and endometrial cancer cell lines exhibiting microsatellite instability. J Biol Chem. 1994;269:14367-70.

25. Koi M, Umar A, Chauhan DP, Cherian SP, Carethers JM, Kunkel TA, et al. Human chromosome 3 corrects mismatch repair deficiency and microsatellite instability and reduces $\mathrm{N}$-methyl-N'-nitro-N-nitrosoguanidine tolerance in colon tumor cells with homozygous hMLH1 mutation. Cancer Res. 1994:54:4308-12
26. Lehar J, Stockwell BR, Giaever G, Nislow C. Combination chemical genetics. Nat Chem Biol. 2008:4:674-81.

27. Ottini L, Falchetti M, Saieva C, De Marco M, Masala G, Zanna I, et al. MRE11 expression is impaired in gastric cancer with microsatellite instability. Carcinogenesis. 2004;25:2337-43.

28. Vilar E, Bartnik CM, Stenzel SL, Raskin L, Ahn J, Moreno V, et al. MRE11 deficiency increases sensitivity to poly(ADP-ribose) polymerase inhibition in microsatellite unstable colorectal cancers. Cancer Res. 2011;71:2632-42.

29. Gaymes TJ, Mohamedali AM, Patterson M, Matto N, Smith A, Kulasekararaj A, et al. Microsatellite instability induced mutations in DNA repair genes CtIP and MRE11 confer hypersensitivity to poly (ADP-ribose) polymerase inhibitors in myeloid malignancies. Haematologica. 2013;98:1397-406.

30. Sandhu SK, Schelman WR, Wilding G, Moreno V, Baird RD, Miranda S, et al. The poly(ADP-ribose) polymerase inhibitor niraparib (MK4827) in BRCA mutation carriers and patients with sporadic cancer: a phase 1 dose-escalation trial. Lancet Oncol. 2013;14:882-92.

31. Clark CC, Weitzel JN, O'Connor TR. Enhancement of synthetic lethality via combinations of ABT-888, a PARP inhibitor, and carboplatin in vitro and in vivo using BRCA1 and BRCA2 isogenic models. Mol Cancer Ther. 2012;11:1948-58

32. Huggins-Puhalla SL, Beumer JH, Appleman JL, Tawbi HA-H, Stoller RG, Lin Y, et al. A phase I study of chronically dosed, single-agent veliparib (ABT-888) in patients (pts) with either BRCA 1/2-mutated cancer (BRCA+), platinum-refractory ovarian cancer, or basal-like breast cancer (BRCA-wt) [abstract]. ASCO. 2012;30:s3054.

\section{Submit your next manuscript to BioMed Central and take full advantage of:}

- Convenient online submission

- Thorough peer review

- No space constraints or color figure charges

- Immediate publication on acceptance

- Inclusion in PubMed, CAS, Scopus and Google Scholar

- Research which is freely available for redistribution 